\title{
Second Births to Teenage Mothers: Risk Factors For Low Birth Weight and Preterm Birth
}

CONTEXT: Teenagers are more likely than older women to have a low-birth-weight infant or a preterm birth, and the risks may be particularly high when they have a second birth. Identifying predictors of these outcomes in second teenage births is essential for developing preventive strategies.

METHODS: Birth certificate data for 1993-2002 were linked to identify second births to Milwaukee teenagers. Predictors of having a low-birth-weight second infant or a preterm second birth were identified using logistic regression.

RESULTS: The same proportion of first and second infants were low-birth-weight (12\%), but second births were more likely than first births to be preterm (15\% vs. 12\%). In analyses that adjusted for demographic, pregnancy and behavioral characteristics, the odds that a second infant was low-birth-weight or preterm were elevated if the mother smoked during pregnancy (odds ratios, 2.2 and 1.9, respectively), had inadequate prenatal weight gain (1.8 and 1.4), had an interpregnancy interval of less than 18 months (1.6-2.9 and 1.4-2.3) or was black (2.7 and 1.7). Women who had received an adequate level of prenatal care had reduced odds of both outcomes (0.6 and 0.4). Women younger than 16 also had increased odds of having a low-birth-weight second infant. Further adjustment for socioeconomic characteristics yielded largely the same results. In addition, women who were unmarried or did not identify a father were at increased risk of both outcomes (1.5 for each), and poor women were at risk of having a low-birth-weight infant (1.3).

CONCLUSIONS: Predictors of poor birth outcomes include modifiable behaviors. Prenatal interventions addressing these behaviors could help improve outcomes.

Perspectives on Sexual and Reproductive Health, 2009, 41(2):101-109, doi: 10.1363/4110109

Teenage pregnancy and childbearing are significant public health problems in most large metropolitan areas of the United States, and rates in Milwaukee are among the highest in the country. ${ }^{1}$ The literature generally shows that the risk of adverse birth outcomes-low or very low birth weight, preterm birth and neonatal death-is greater for teenagers than for older women. ${ }^{2,3}$ However, studies disagree about what risk factors are associated with these adverse outcomes and whether young maternal age poses an increased risk when other factors are controlled for. ${ }^{2,4}$ Other maternal characteristics that have been examined as potential explanatory variables for increased risk of adverse outcomes in teenage births are low socioeconomic status and minority race or ethnicity. ${ }^{3,5}$ A number of behavioral and medical risk factors have also been associated with adverse birth outcomes in teenagers: lack of appropriate prenatal care or weight gain, smoking, alcohol use, illicit drug use and sexual risk-taking., ${ }^{4,6}$

A second teenage birth may be even more deleterious to mother and child than a single teenage birth because of compounded socioeconomic impacts and the influence of short interpregnancy intervals. ${ }^{7}$ Women with interpregnancy intervals of less than 18 months appear to be at greater risk of having a premature birth or a low-birth-weight baby than women with longer intervals. ${ }^{8}$ However, a number of studies that examined outcomes in higher order births to teenagers found preterm birth to be consistently associated with higher parity, while others indicate no increased risk of preterm birth or other adverse outcomes. ${ }^{9,10}$ Teenage childbearing has a negative impact on educational attainment, which can lead to long-term economic disadvantage. ${ }^{11}$ Teenage mothers who have a second birth within two years are less likely to complete high school than are those who postpone a second pregnancy. ${ }^{12}$

A systematic review of the literature found that $20-37 \%$ of teenage mothers had a second birth within 24 months. ${ }^{13}$ In a review of programs that targeted pregnant teenagers or teenage mothers, Klerman found that most programs were not able to reduce the proportion of participants who had an additional birth within 24 months to below 20-25\%. ${ }^{7}$ The most recent national birth data reported that 81,517 teenage births (19\%) in 2005 were second or higher order births. ${ }^{14}$ Even though this number represents a decrease from previous years, it is still substantial. ${ }^{15}$ Additionally, preliminary data indicate that the number of repeat teenage births rose to almost 85,000 in $2006 .{ }^{16}$

The ability to identify births to the same woman is necessary to understand the poor health outcomes associated with repeat childbearing. Many birth databases contain information on previous live births, but do not link births to the same mother. Thus, it is often possible to look at characteristics and outcomes of first and second births, but
By Susan N.

Partington,

Dale L. Steber,

Kathleen A. Blair

and Ron A. Cisler

Susan N. Partington is assistant professor, and Ron A. Cisler is associate professior, both in the Department of Health Sciences, College of Health Sciences, University of Wisconsin, Milwaukee; Dale L. Steber is supervisor, information technology, Center for Urban Population Health, Aurora Health Care, Milwaukee; and Kathleen A. Blair is epidemiologist, City of Milwaukee Health Department. 
not first and second births to the same mother. Examining data for the same mother provides control for biological factors that vary among women.

In the study described here, we used record linkage to identify repeat births to Milwaukee teenagers during the period 1993-2002, and assessed characteristics associated with adverse second-birth outcomes. The study was approved by the University of Wisconsin Health Sciences Institutional Review Board.

\section{METHODS}

Background

Milwaukee is the largest metropolitan area in the state; its 596,974 residents represent $11 \%$ of the total state population. ${ }^{17}$ According to the 2000 census, $45 \%$ of the population was non-Hispanic white, $37 \%$ non-Hispanic black and $12 \%$ Hispanic. ${ }^{17}$ The median household income in 1999 was $\$ 32,216$, and $21 \%$ of residents for whom poverty status was determined had family incomes below the poverty level. ${ }^{17}$ In 2004, some $19 \%$ of teenage births in Wisconsin were repeat births, compared with 20\% nationally; ${ }^{18}$ in Milwaukee, $25 \%$ of teenage births were repeat births. ${ }^{19}$ During the study period, mandatory birth certificate data reported by hospitals to the Wisconsin Office of Vital Records recorded 111,862 births.

To identify repeat births, it was necessary to find records of births to the same mother and create a family or sibling structure-that is, to identify related births and establish their order. The birth certificate records in the master birth file held by the Milwaukee health department did not contain any unique maternal identifiers. Therefore, as is common when unique identifiers are not available, we employed a probabilistic matching procedure to link records. ${ }^{20}$ The method works well with the kinds of errors often found in large data sets (e.g., misspellings, data entry errors, inconsistent use of abbreviations and missing data), because an exact match is not required.

\section{Record Linkage Procedure}

- Creation of birth record database. Birth certificate data for 1993-2002 existed in 11 files in four formats with a variety of field names and coding schemes. The data were consolidated and were extensively cleaned, recoded and reformatted where necessary to reconcile differences among the original files. This step, using Microsoft Access 2000, resulted in the creation of a master database, containing 111,862 birth records, and a data dictionary. A unique identification number was assigned to each birth record. The master file was then duplicated for the probabilistic match.

-Identifying potential matches. The duplicate birth files were matched against each other in FEBRL (Freely Extensible Biomedical Record Linkage), version 0.2.2. ${ }^{21}$ To identify potential matches, FEBRL employs user-selected comparators to examine the same data element in two records. A similarity value is assigned on the basis of this examination. Similarity values are summed for each record pair to produce a final weight, which is used to determine if the record pair is a match.

Prior to performing the record linkage, we ran numerous match routines in FEBRL, using random samples from the birth file, and reviewed them manually until the most accurate comparison method for the data was determined. On the basis of this information, we used three comparators to compensate for typographical errors and an exact comparator to identify identical data elements. The comparators examined mothers' first, middle, last and maiden names; state, day, month and year of birth; and race. Street address and husband's name, if available, were also included. The results of the random file matches also provided information to set upper and lower threshold weights. Record pairs with weights above the upper threshold were designated matches, and those with weights below the lower threshold were nonmatches.

Additionally, before performing the linkage, we used a standardizer to minimize the effect of differences in capitalizations, spacing and abbreviations in fields. The correction lists used by the FEBRL standardizer were modified to address specific problems in the birth certificate data set.

After standardizing, comparing and classifying records, FEBRL produced an output file that listed every record in one birth certificate file and the possible matches in the comparison file that were above the lower threshold. The record in the comparison file that produced the largest weight was designated the matching record. The assigned match was ignored because the birth file was compared with itself, and the most highly weighted pair would have been the same birth record from each file. In addition, the primary goal was to identify families, or groups of matched records, not single matches. FEBRL was modified to produce an additional output file that included the unique birth record identification number for each record of every pair produced and the accompanying weight.

The match process produced a total of 261,920 record pairs, of which $81 \%$ were above the match threshold; $13 \%$ were below the lower threshold, and were considered true nonmatches. A manual review was necessary for $7 \%$ of the potential matches.

- Manual review of possible matches. FEBRL produced a birth details output file that displayed data fields for each potential matched pair side by side. Two reviewers used established criteria to determine whether each pair was a match. First, they compared the mother's first, middle, last and maiden names and date of birth; if a change in last name could be attributed to marriage or if other differences could be attributed to obvious typographic errors or character transpositions, the pair were scored as a match. The reviewers then compared infants' birth dates on the two records to confirm that it was physiologically possible for the infants to have the same mother; if the dates were less than six months apart, the pair were scored as a nonmatch. 
-Family structure created, and family number assigned. When the manual review was completed, all record pairs and accompanying weights were loaded into Microsoft Access. Duplicate record pairs, those with weights below the lower threshold and those that the manual review determined were nonmatches were eliminated. The remaining unique record pairs made up the match database. A Java program was written to create a family structure and assign a family number. This program compared each record pair to the others in the match database. A record in any pair that was also in the first pair was added to the family of the first pair and assigned the same family number. The process was repeated until all records in the file had been allocated to a family. The result was a list of birth record identification numbers and an associated family number for families with more than one birth. Families with a single birth were assigned family numbers sequentially. This file was merged with the master birth file, using the birth record identification number as the unique identifier.

-Manual review of large families. When family number had been assigned, families with more than six members were again reviewed manually to confirm correct family assignment, and incorrect placements were reassigned. At this point, all identifiers in the data except geographic coordinates were removed.

-Event order and birth order assigned. Once an accurate family structure had been built, Java programs were written to assign birth order and birth event order within families, to distinguish between deliveries and infants within families. A birth event was defined as a single delivery; both singleton and multiple births were considered one birth event. Birth order was assigned on the basis of the infant's birth date. Each record of a multiple birth was assigned the same birth event number. Additionally, the birth event program was written to differentiate between a first and only birth and a first of more than one birth per family. Birth order and birth event were then added to the master birth database. With birth event order in place, it was possible to identify repeat births to teenagers.

\section{Analysis}

Teenage births were defined as births to females younger than 20. The birth certificate data set contained few maternal individual-level data elements (only age, address at time of birth, highest grade completed, a text description of occupation, marital status and maternal birth state). Most of the data were related to the infant and birth outcome. There was no family information, and most records did not contain paternal information. The master birth file contained accurate geographic location coordinates (geocodes) for $95 \%$ of the births in the file. Geocodes from the mother's address at the time of birth were used to determine block group of residence. Median household income was determined from 2000 census data by block group of residence for each birth. ${ }^{22}$ (A block group is a subdivision of a census tract and usually contains 600-3,000 people; optimal size is 1,500 people. ${ }^{23}$ It is the smallest geographic area for which the census publishes sample data. ${ }^{24}$ )

The 10 years of data contained records on 22,660 births to 18,050 teenagers. Of these births, 14,451 were first and only ones, 3,761 were the first of more than one, 3,784 were second births, 602 were third births, and 62 were fourth or fifth ones. Preterm delivery and low birth weight occur more often with multiple than with singleton births. ${ }^{25}$ Therefore, if the first or second teenage birth was a multiple, infants from both birth events were excluded from the analysis. Infants born at less than 20 weeks' gestation were also excluded. In all, 215 births were excluded (96 first and 119 second teenage births), resulting in a sample of 3,665 birth pairs.

Chi-square tests were used to compare first and second, or repeat, teenage births by selected outcomes and selected characteristics of the mother and pregnancy, as reported on the birth certificates. Low birth weight was defined as less than 2,500 g at birth, and very low birth weight as less than 1,500 g. ${ }^{26}$ Preterm births were those that occurred prior to 37 weeks' gestation. ${ }^{27}$ Interpregnancy interval was calculated by subtracting the date of the first teenage birth from the date of the second, and then subtracting the second infant's gestational age, as estimated on the birth certificate. The amount of prenatal weight gain would be expected to be related to gestational agethat is, women who deliver early have less opportunity for weight gain. ${ }^{25}$ Rate of prenatal weight gain was calculated by dividing total weight gained or lost during the pregnancy by gestational age, and then was dichotomized into a gain of less than 0.6 pounds per week (characterized as inadequate) or 0.6 or more pounds per week (adequate), based on rate of net gain associated with decreased risk of preterm birth. ${ }^{28}$ Adequacy of prenatal care was computed using the Adequacy of Prenatal Care Index, which takes into account timing of first prenatal visit and number of such visits. ${ }^{29}$

Unadjusted odds ratios were computed to assess relationships between a second infant's low birth weight or preterm birth and maternal demographic characteristics (race, ethnicity and age), socioeconomic characteristics (median household income of census block group, paternity or marital status* and completed education), and pregnancy and behavioral characteristics (prenatal weight gain, previous adverse birth outcome, smoking during pregnancy, prenatal care utilization and interpregnancy interval). Logistic regression was used to calculate adjusted odds ratios. Two regression models were constructed: The first included only demographic and pregnancy or behavioral characteristics; the second added socioeconomic indicators.

*In Wisconsin, if a mother is married, her husband must be recorded on the birth certificate, even if he is not the father. If a mother is not married, paternity may be established by a voluntary acknowledgment of paternity, or it can be adjudicated by court order. In the last two situations, the father can be added to the record at any time after birth, making relevance to the pregnancy uncertain. 
TABLE 1. Percentage distribution of first and second births to the same teenage mother, by selected maternal characteristics and birth outcomes, Milwaukee 1993-2002

Characteristic
or outcome

First birth Second birth

DEMOGRAPHIC

Race/ethnicity

White

Black

Hispanic

Other

Age

$<16$

16

17

18

19

SOCIOECONOMIC

Completed education (yrs.)***, $\dagger$

$\geq 12$

$\leq 11$

Marital/paternity status***

Married or paternity established

Single, paternity not established

Household income (1999)†

$\leq \$ 10,000$

$\$ 10,000-19,999$

$\$ 20,000-29,999$

$\$ 30,000-39,999$

$\geq \$ 40,000$

PREGNANCY/BEHAVIORAL

Weekly prenatal weight gain***,

Adequate

Inadequate

Prenatal care utilization ${ }^{* * *}, \dagger$

Inadequate

Intermediate

Adequate

$>$ adequate

Smoked during pregnancy***

Yes

No

Interpregnancy interval (mos.)

$<3$

$3-5$

6-11

12-17

$\geq 18$

BIRTH OUTCOME

Low birth weight

Yes

No

12.0

11.5

Very low birth weight

Yes

No

2.4

97.6

2.6

Preterm**

Yes

No

Total

12.4

14.8

100.0

${ }^{* *} \mathrm{p}<.01 .{ }^{* * *} \mathrm{p}<.001$. Includes some imputed values. Notes: na=not applicable. Data include only singleton births. Inadequate prenatal weight gain is a net gain of less than 0.6 pounds per week. Adequacy of prenatal care was measured on a standard scale and takes into account timing of first visit and number of visits. Infants were low-birth-weight if they weighed less than $2,500 \mathrm{~g}$ at birth; they were very low birth weight if they weighed less than

$1,500 \mathrm{~g}$. Preterm birth was a birth at less than 37 weeks' gestation.

\section{Missing Data}

Nine percent of records were missing data on weight gain during pregnancy, and $2 \%$ were missing the neighborhood poverty indicator because addresses could not be assigned a geocode; very small numbers of records were missing data on prenatal care utilization and mother's education. Missing value analysis indicated that these values were not missing completely at random. As a result, we replaced missing values with imputed values instead of dropping these cases from the analysis. Multiple imputed values were generated using NORM, version $2 .{ }^{30}$ Five sets of imputed data were created and analyzed. The results were entered back into NORM and combined to generate average parameter estimates, pooled standard errors and $\mathrm{p}$ values for the estimates. ${ }^{31}$ The average beta coefficients were used to compute odds ratios. Chi-square statistics were calculated from average counts of the five imputed data sets between first and second births. Stata/MP, version 10.0, was used for all analyses.

\section{RESULTS}

At the time of the first birth, $29 \%$ of teenage mothers were younger than $16,28 \%$ were 16 years old, 28\% were 17 years old, and only $15 \%$ were 18 or older (Table 1). By the second birth, $24 \%$ of teenage mothers had completed 12 years of schooling; however, only 19\% had finished high school between births. More of these young women were married or had paternity established at their second infant's birth than at their first (41\% vs. $25 \%$ ), but $60 \%$ were still single and had not established paternity. The distribution of median household income did not change between the first and second teenage birth.

The proportion of mothers who had inadequate weight gain during pregnancy increased between the first and second births (49\% and 61\%, respectively), as did the proportions who received inadequate prenatal care (30\% vs. $37 \%$ ) and who smoked while pregnant (10\% vs. 19\%). Pregnancies were at least 18 months apart for $31 \%$ of mothers, 6-17 months apart for 46\% and fewer than six months apart for $23 \%$. The prevalence of low birth weight and very low birth weight did not change (12\% and 2-3\%, respectively), but the second birth was more likely than the first to be preterm (15\% vs. $12 \%$ ).

In unadjusted analyses, both the odds that a teenager's second infant was low-birth-weight and the odds that the second birth was preterm were associated with a wide range of characteristics (Table 2). These included the mother's being 16 or younger (odds ratios, 1.9-3.0), having had inadequate prenatal weight gain (1.4-1.8), having had a previous adverse birth outcome (3.8-4.5), smoking during pregnancy (1.6-1.8) and having had any interpregnancy interval of less than 18 months (1.3-2.7). Compared with women who had inadequate prenatal care, those who had intermediate or adequate care had lower odds of both outcomes (0.3-0.6), and those who had more than adequate care had higher odds of both (2.8-3.1). Women who were black, were 18 years old or were in the lower half of the 


\begin{tabular}{|c|c|c|c|c|c|c|}
\hline \multirow[t]{3}{*}{ Characteristic } & \multicolumn{3}{|c|}{ Low birth weight } & \multicolumn{3}{|l|}{ Preterm } \\
\hline & \multirow[t]{2}{*}{ Unadjusted } & \multicolumn{2}{|l|}{ Adjusted } & \multirow[t]{2}{*}{ Unadjusted } & \multicolumn{2}{|l|}{ Adjusted } \\
\hline & & Model 1 & Model 2 & & Model 1 & Model 2 \\
\hline \multicolumn{7}{|l|}{$\begin{array}{l}\text { DEMOGRAPHIC } \\
\text { Race/ethnicity }\end{array}$} \\
\hline White (ref) & 1.00 & 1.00 & 1.00 & 1.00 & 1.00 & 1.00 \\
\hline Black & $2.06^{* *}$ & $2.73^{* * *}$ & $2.29^{* *}$ & 1.31 & $1.66^{* *}$ & 1.43 \\
\hline Hispanic & 1.15 & 1.39 & 1.29 & 0.89 & 1.06 & 1.00 \\
\hline Other & 1.01 & 1.19 & 1.19 & 0.72 & 0.84 & 0.87 \\
\hline \multicolumn{7}{|l|}{ Age } \\
\hline 19 (ref) & 1.00 & 1.00 & 1.00 & 1.00 & 1.00 & 1.00 \\
\hline$<16$ & $3.04^{* * *}$ & $2.67^{* *}$ & $2.35^{*}$ & $2.53^{* *}$ & $2.05^{*}$ & 1.76 \\
\hline 16 & $2.22^{* * *}$ & $1.58^{*}$ & 1.39 & $1.89^{* * *}$ & 1.34 & 1.17 \\
\hline 17 & 1.17 & 0.94 & 0.87 & 1.13 & 0.93 & 0.85 \\
\hline 18 & $1.31^{*}$ & 1.18 & 1.15 & 1.23 & 1.13 & 1.08 \\
\hline \multicolumn{7}{|l|}{ PREGNANCY/BEHAVIORAL } \\
\hline \multicolumn{7}{|l|}{ Weekly prenatal weight gaint } \\
\hline Adequate (ref) & 1.00 & 1.00 & 1.00 & 1.00 & 1.00 & 1.00 \\
\hline Inadequate & $1.81^{* * *}$ & $1.82^{* * *}$ & $1.79 * * *$ & $1.44^{*}$ & $1.43^{* *}$ & $1.40^{* *}$ \\
\hline \multicolumn{7}{|l|}{ Previous adverse birth outcome } \\
\hline None (ref) & 1.00 & 1.00 & 1.00 & 1.00 & 1.00 & 1.00 \\
\hline Low birth weight or preterm & $4.47^{* * *}$ & $3.84^{* * *}$ & $3.84^{* * *}$ & $3.84^{* * *}$ & $3.27^{* * *}$ & $3.27^{* * *}$ \\
\hline \multicolumn{7}{|l|}{ Prenatal care utilization† } \\
\hline Inadequate (ref) & 1.00 & 1.00 & 1.00 & 1.00 & 1.00 & 1.00 \\
\hline Intermediate & $0.56^{* *}$ & 0.71 & 0.69 & $0.46^{* * *}$ & $0.55^{* * *}$ & $0.54^{* * *}$ \\
\hline Adequate & $0.48^{* * *}$ & $0.64^{* *}$ & $0.66^{* *}$ & $0.34^{* * *}$ & $0.41^{* * *}$ & $0.42^{* * *}$ \\
\hline >adequate & $2.84^{* * *}$ & $3.60^{* * *}$ & $3.83^{* * *}$ & $3.14^{* * *}$ & $3.69^{* * *}$ & $3.95^{* * *}$ \\
\hline \multicolumn{7}{|l|}{ Smoked during pregnancy } \\
\hline No (ref) & 1.00 & 1.00 & 1.00 & 1.00 & 1.00 & 1.00 \\
\hline Yes & $1.84^{* * *}$ & $2.24^{* * *}$ & $2.11^{* * *}$ & $1.60^{* * *}$ & $1.85^{* * *}$ & $1.74^{* * *}$ \\
\hline \multicolumn{7}{|l|}{ Interpregnancy interval (mos.) } \\
\hline$\geq 18$ (ref) & 1.00 & 1.00 & 1.00 & 1.00 & 1.00 & 1.00 \\
\hline$<3$ & $2.66^{* * *}$ & $2.91^{* * *}$ & $3.04^{* * *}$ & $1.90^{* * *}$ & $1.94^{* *}$ & $2.04^{* * *}$ \\
\hline $3-5$ & $1.93^{* * *}$ & $2.00 * * *$ & $2.02^{* * *}$ & $2.16^{* * *}$ & $2.31^{* * *}$ & $2.36^{* * *}$ \\
\hline $6-11$ & $1.44^{*}$ & $1.56^{* *}$ & $1.54^{*}$ & $1.32^{*}$ & $1.40 *$ & $1.39 *$ \\
\hline $12-17$ & $1.49^{*}$ & $1.63^{*}$ & $1.63^{*}$ & $1.40^{*}$ & $1.53^{* *}$ & $1.54^{* *}$ \\
\hline \multicolumn{7}{|l|}{ SOCIOECONOMIC } \\
\hline \multicolumn{7}{|l|}{ Completed education (yrs.)† } \\
\hline$\geq 12$ (ref) & 1.00 & na & 1.00 & 1.00 & na & 1.00 \\
\hline$\leq 11$ & $1.37^{*}$ & na & 1.08 & $1.31^{*}$ & na & 1.13 \\
\hline \multicolumn{7}{|l|}{ Marital/paternity status } \\
\hline Married or paternity established (ref) & 1.00 & na & 1.00 & 1.00 & na & 1.00 \\
\hline Single, paternity not established & $1.70^{* * *}$ & na & $1.47^{* *}$ & $1.59^{* * *}$ & na & $1.52^{* * *}$ \\
\hline \multicolumn{7}{|l|}{ Household income (1999)† } \\
\hline$>50$ th percentile (ref) & 1.00 & na & 1.00 & 1.00 & na & 1.00 \\
\hline$\leq 50$ th percentile & $1.41^{* *}$ & na & $1.28^{*}$ & 1.23 & na & 1.19 \\
\hline
\end{tabular}

income distribution had elevated odds of having a lowbirth-weight second infant, but not of preterm delivery.

After adjustment for demographic, pregnancy and behavioral characteristics (adjusted model 1), results were largely unchanged. The odds of both having a low-birthweight second infant and having a preterm second birth remained positively associated with inadequate weight gain (odds ratios, 1.8 and 1.4, respectively), more than adequate prenatal care (3.6 and 3.7), previous poor birth outcome (3.8 and 3.3), smoking during pregnancy (2.2 and 1.9) and all interpregnancy intervals less than 18 months (1.6-2.9 and 1.4-2.3). In the adjusted analysis, black women had elevated odds of both having a low-birthweight infant and having a preterm birth (2.7 and 1.7, respectively). Mothers who were younger than 16 still had an increased likelihood of both outcomes (2.7 and 2.1), 
but 16-year-olds were at increased risk only of having a low-birth-weight baby (1.6). Adequate prenatal care remained negatively associated with the odds of both outcomes ( 0.6 for low birth weight and 0.4 for preterm birth), but intermediate care was associated only with the likelihood of preterm birth (0.6).

Adding socioeconomic indicators did not appreciably change the results for the pregnancy and behavioral characteristics, or for most demographic characteristics. However, mothers who were 16 years old no longer had an increased risk of having a low-birth-weight baby, and blacks and mothers younger than 16 no longer had elevated odds of preterm birth. In addition, the odds of Health risk low birth weight and preterm birth were raised if no father behaviors

\section{during}

pregnancy...

were more

common

beforea

second birth

than beforea

first birth.

was listed on the birth record (odds ratio, 1.5 for each); income below the 50th percentile was associated with increased odds of low birth weight (1.3).

\section{DISCUSSION}

Our study extends previous research on repeat teenage childbearing by comparing births to the same mothers. This approach permitted the mother to serve as a control for biological factors and medical history that may have influenced birth outcomes. With this control in place, we found that a significantly greater proportion of second than of first teenage births were preterm; the prevalence of low birth weight did not differ between first and second babies born to teenagers. Additionally, health risk behaviors during pregnancy (inadequate weight gain, inadequate level of prenatal care and smoking) were more common before a second birth than before a first birth, and the elevated risk of adverse birth outcomes associated with these behaviors was not substantially changed by controlling for the effects of demographic and socioeconomic characteristics.

Interpregnancy intervals of less than 18 months have been associated with a number of poor obstetric outcomes, including low birth weight and preterm birth. For example, Zhu found the lowest risk of these outcomes associated with interpregnancy intervals of 18 months. ${ }^{8}$ We found a similar trend: Shorter intervals were associated with significantly increased odds of low birth weight and preterm birth.

In previous research, lack of early and consistent prenatal care has been associated with both low birth weight ${ }^{32}$ and preterm birth, ${ }^{33}$ and teenagers who have had a previous birth have been shown to have a reduced likelihood of initiating early prenatal care and an elevated likelihood of receiving no prenatal care. ${ }^{34}$ In our sample, an adequate level of prenatal care before the second teenage birth was associated with reduced odds of both low birth weight and preterm birth when compared with inadequate care. However, teenagers who had received more than adequate prenatal care had substantially increased odds of having a low-birth-weight second baby and a preterm second birth. A similar association between more than adequate care and low birth weight has been noted previously. ${ }^{35}$ Perhaps teenagers with more than adequate care represented a group who were recognized as high-risk and who thus made more and earlier prenatal care visits.

Although paternity and marital status may reflect different situations, second infants born to women who had not established paternity and were not married (according to the birth certificate) were more likely than others to be low-birth-weight or preterm. These findings are consistent with those of earlier work: Gaudino and colleagues found that nonreporting of the father on the birth certificate was associated with increased risks of low birth weight and infant mortality ${ }^{36}$ and Luo et al. found that single mothers had higher odds of adverse pregnancy outcomes than mothers in legal or common-law marriages. ${ }^{37}$ Marriage or established paternity may serve as a proxy for increased family income, as well as increased psychosocial support during pregnancy, and may thereby contribute to improved outcomes.

Smoking during the pregnancy preceding a second birth was associated with increased odds of both low birth weight and preterm birth even after demographic, socioeconomic, pregnancy and behavioral characteristics were controlled for. Smoking during pregnancy has been consistently associated with decreased birth weight and preterm birth in both teenage and older mothers. ${ }^{38,39}$ One study estimated that among infants born to teenagers, maternal smoking during pregnancy accounted for 39\% of the risk of low birth weight and $12 \%$ of the risk of preterm birth; for infants born to adults, the figures were 28\% and 19\%, respectively. ${ }^{40}$ These data suggest that smoking cessation and prevention in pregnancy may be even more crucial for teenagers than for adults.

The number of teenagers who reported smoking during the pregnancy preceding their second birth was almost twice the number who said they smoked during the pregnancy leading to the first birth. From 1990 to 2000, the proportion of women who smoked during pregnancy declined nationwide, but it was highest among 18-19year-olds. ${ }^{41}$ Smoking during pregnancy may be underreported on birth certificates, but trends and variations we observed are similar to those seen in other national data sources. ${ }^{41}$

Nationwide, a higher proportion of infants born to black teenagers than to white or Hispanic teenagers are preterm or low-birth-weight. ${ }^{14}$ Studies that have examined the contributions of race and ethnicity to adverse birth outcomes among teenagers have produced varied results. Chen et al. found that teenagers had higher risks than 20-24year-olds of having preterm and low-birth-weight infants, regardless of race or ethnicity. ${ }^{2}$ DuPlessis and colleagues found, in analyses controlling for socioeconomic indicators, that black teenagers were significantly more likely than whites to have a preterm birth or a low-birth-weight infant. ${ }^{42}$ In our sample, when socioeconomic indicators were added to the models, an association between being black and the risk of having a preterm birth disappeared, although the risk of black women's having a low-birthweight infant remained significantly elevated. 
Prior studies have also associated the level of maternal education and neighborhood socioeconomic indicators with adverse birth outcomes, particularly among black women. ${ }^{43,44}$ In our final model, neighborhood poverty (median household income from block group) was associated only with low birth weight, and educational level was not associated with either outcome. The lack of variation in educational attainment, due to the limited age span studied, may account for this finding.

Significantly more of the teenage mothers in our sample had an inadequate weight gain during the second pregnancy than during the first; consistent with findings from earlier research, this characteristic was associated with increased odds of both having a low-birth-weight infant ${ }^{45}$ and delivering preterm. ${ }^{46}$ Teenagers' energy and nutrient needs during pregnancy are generally greater than those of adult women, because of the ongoing needs for growth and development of the teenage mother in addition to the needs of the fetus. ${ }^{47}$ The enhanced need for nutrients, coupled with the poor dietary patterns and food choices typical among teenagers, especially low-income teenagers, contributes to teenagers' inadequate weight gain and suboptimal nutrient intake during pregnancy. ${ }^{48}$

Previous reports have concluded that closely spaced teenage births make finishing high school and finding employment difficult, resulting in poverty and reliance on welfare. ${ }^{12}$ Although more teenage mothers in our sample had completed 12 years of schooling at the second birth than at the first, most were not high school graduates. Additionally, the majority were still single parents. Median household income changed very little between first and second teenage births. Educational attainment and paternity status improved; however, most of these repeat teenage mothers were at much the same place economically, educationally and socially, with the additional burden of another child.

\section{Limitations}

The study is limited by potential record mismatches and, therefore, misclassification of births. However, the most significant limitations are those inherent in using vital records data. Birth certificate data do not include measures of a number of potential confounding factors, including maternal anthropometry and nutritional status, paternal information (demographic, socioeconomic and anthropometric characteristics), individual-level income, stress level, level of family and other social supports, and illegal substance use. Although birth records of women who lived in Milwaukee but delivered elsewhere in the state were included in the database, some teenagers likely had a first birth in other states and a second birth in Milwaukee; as a result, the actual number of repeat teenage pregnancies was likely greater than is reported here. Inaccuracies in reporting and recording of information also are likely sources of error in birth data. The imputed values used for missing data may not accurately reflect the missing data. However, dropping cases with missing data may have resulted in bias if they were systematically different from cases with complete data. Complete case analyses produced very similar results to those produced with imputed values. Additionally, our results do not show causality, only statistical associations.

Finally, the increase we observed in prenatal smoking may reflect that younger mothers underreport smoking, to avoid disapproval of parents or health professionals. However, underreporting of prenatal smoking on birth certificates does not appear to be age-related. ${ }^{41}$

\section{Implications}

The persistence of inadequate prenatal care utilization and prenatal weight gain, teenage mothers' short interpregnancy intervals, their increase in smoking during pregnancy and the magnitude of the odds ratios for adverse birth outcomes associated with these behaviors provide evidence of the importance of identifying and addressing modifiable health risk behaviors during routine clinic visits. Previous research has established that providing prenatal health behavior education and advice reduces the likelihood of both low birth weight and preterm birth. ${ }^{49,50}$ Regular prenatal visits provide an opportunity to deliver educational interventions to a receptive audience. A number of methods to deliver behavioral counseling in a health care setting have been found cost-effective and efficacious, including brief interventions and motivational interviewing. ${ }^{48,51,52}$ Consistent, thorough screening for each of the above-mentioned behaviors at the first prenatal visit, followed by counseling and reassessment, reinforcement and encouragement at subsequent visits or by telephone, could promote behavior change and improve outcomes.

Barriers to access and underutilization of prenatal care, as well as underutilization of the Special Supplemental Nutrition Program for Women, Infants, and Children (WIC), have also been identified as contributors to adverse birth outcomes for teenagers. ${ }^{53}$ Strategies to address utilization issues that may be effective for teenagers include text messaging of appointment reminders, priority appointment scheduling, resolving transportation difficulties and providing access to resources, such as WIC and Medicaid. Additionally, stress has been identified as a significant barrier to success in smoking cessation, particularly in low-income women. ${ }^{54}$ A teenage mother pregnant for the second time would likely benefit from counseling on relaxation techniques and stress management in general, and this may be particularly important for smokers.

Educational status was not associated with low birth weight and preterm birth in this population, but most of these young mothers were not high school graduates. Teenage mothers are frequently from disadvantaged backgrounds, and inadequate educational attainment contributes to continued poverty for their predominantly single-parent families. Programs that encourage and support young mothers in finishing high school and pursuing higher education are essential for the long-term economic well-being of both the mother and her children. 


\section{REFERENCES}

1. Ikramullah E et al., Facts at a glance: a fact sheet reporting national, state-level, and city-level trends in teen childbearing, 2007, Child Trends, <http://www.childtrends.org/Files/Child_Trends-06_ 26_2007_FG_2007FactsAtAGlance.pdf>, accessed Apr. 7, 2008.

2. Chen XK et al., Teenage pregnancy and adverse birth outcomes: a large population based retrospective cohort study, International Journal of Epidemiology, 2007, 36(2):368-373.

3. Gilbert $\mathrm{W}$ et al., Birth outcomes in teenage pregnancies, Journal of Maternal-Fetal Medicine, 2004, 16(5):265-270.

4. Strobino DM et al., Mechanisms for maternal age differences in birth weight, American Journal of Epidemiology, 1995, 142(5):504514.

5. Reichman NE and Pagnini DL, Maternal age and birth outcomes: data from New Jersey, Family Planning Perspectives, 1997, 29(6): 268-272 \& 295.

6. Kaiser MM and Hays BJ, Health-risk behaviors in a sample of first-time pregnant adolescents, Public Health Nursing, 2005, 22(6): 483-493.

7. Klerman JA, Another Chance: Preventing Additional Births to Teen Mothers, Washington, DC: The National Campaign to Prevent Teen Pregnancy, 2004

8. Zhu B-P, Effect of interpregnancy interval on birth outcomes: findings from three recent US studies, International Journal of Gynaecology E Obstetrics, 2005, 89(Suppl. 1):525-533.

9. Klerman LV, Risk of poor pregnancy outcomes: is it higher among multiparous teenage mothers? Journal of Adolescent Health, 2006, 38(6):761-764.

10. Akinbami LJ et al., Risk of preterm birth in multiparous teenagers, Archives of Pediatrics and Adolescent Medicine, 2000, 154(11): 101-107.

11. Hofferth SL et al., The effects of early childbearing on schooling over time, Family Planning Perspectives, 2001, 33(6):259-267.

12. Polit DF and Kahn JR, Early subsequent pregnancy among economically disadvantaged teenage mothers, American Journal of Public Health, 1986, 76(2):167-171

13. Meade CS and Ickovics JR, Systematic review of sexual risk among pregnant and mothering teens in the USA: pregnancy as an opportunity for integrated prevention of STD and repeat pregnancy, Social Science \& Medicine, 2005, 60(4):661-678.

14. Martin JA et al., Births: final data for 2005 , National Vital Statistics Reports, 2007, Vol. 56, No. 6.

15. Ventura SC et al., Births to teenagers in the United States, 1940 2000, National Vital Statistics Reports, 2001, Vol. 49, No. 10.

16. Hamilton BE et al., Births: preliminary data for 2006, National Vital Statistics Reports, 2007, Vol. 56, No. 7.

17. U.S. Bureau of the Census, CENSUS 2000 American Fact Finder, 2005, <http://factfinder.census.gov/home/saff/main.html?_lang=en>, accessed Apr. 5, 2007.

18. Schelar E et al., Repeat teen childbearing: differences across states and by race and ethnicity, Child Trends Research Brief, 2007, Child Trends, <http://www.childtrends.org/Files/Child_Trends-2007_10_ 25_RB_Repeat.pdf>, accessed Feb. 25, 2008.

19. Wisconsin Department of Health and Family Services, WISH query teen births module (percent of births to teens), 2008, <http:// dhfs.wisconsin.gov/wish/measures/teen_brths/long_form.html>, accessed Mar. 11, 2008

20. Clark DE, Practical introduction to record linkage for injury research, Injury Prevention, 2004, 10(3):186-191.

21. Christian P and Churches T, FEBRL (Freely Extensible Biomedical Record Linkage), 0.2.2, Canberra, Australia: Australian National University, 2003.
22. Krieger N, Overcoming the absence of socioeconomic data in medical records: validation and application of a census-based methodology, American Journal of Public Health, 1992, 82(5):703-710.

23. U.S. Bureau of the Census, Census 2000 Geographic Terms and Concepts, 2002, <http://www.census.gov/geo/www/reference.html>, accessed June 26, 2008.

24. U.S. Bureau of the Census, Geographic Areas Reference Manual: Census Blocks and Block Groups, <http://www.census.gov/geo/www/ garm.html>, accessed June 26, 2008.

25. Mathews TJ and MacDorman MF, Infant mortality statistics from the 2004 period linked birth/infant death data set, National Vital Statistics Reports, 2007, Vol. 55, No. 14.

26. Institute of Medicine, Nutrition During Pregnancy, Washington, DC: National Academy Press, 1990

27. Berkowitz GS and Papiernik E, Epidemiology of preterm birth, Epidemiologic Reviews, 1993, 15(2):414-440.

28. Abrams B et al., Maternal weight gain and preterm delivery, $\mathrm{Ob}$ stetrics \& Gynecology, 1989, 74(4):577-583

29. Kotelchuck M, An evaluation of the Kesser adequacy of prenatal care index and a proposed adequacy of prenatal care utilization index, American Journal of Public Health, 1994, 84(9):1414-1420.

30. Schafer JL, NORM: multiple imputation of incomplete multivariate data under a normal model, software for Windows 95/98/NT, 1999, <http://www.stat.psu.edu/ jls/misoftwa.html>, accessed Jan. 20, 2006.

31. Sinharay $\mathrm{S}$ et al., The use of multiple imputation for the analysis of missing data, Psychological Methods, 2001, 6(4):317-329.

32. Alexander GR and Kotelchuck M, Assessing the role and effectiveness of prenatal care: history, challenges, and directions for future research, Public Health Reports, 2001, 116(4):306-316.

33. Vintzileos AM et al., The impact of prenatal care in the United States on preterm births in the presence and absence of antenatal high-risk conditions, American Journal of Obstetrics and Gynecology, 2002, 187(5):1254-1257.

34. Hueston WJ et al., Prenatal care initiation among pregnant teens in the United States: an analysis over 25 years, Journal of Adolescent Health, 2008, 42(3):243-248.

35. Kotelchuck $\mathrm{M}$, The adequacy of prenatal care utilization index: its US distribution and association with low birthweight, American Journal of Public Health, 1994, 84(9):1486-1489.

36. Gaudino JA, Jr., et al., No fathers' names: a risk factor for infant mortality in the state of Georgia, USA, Social Science \& Medicine, 1999, 48(2):253-265.

37. Luo Z-C et al., Disparities in pregnancy outcomes according to marital and cohabitation status, Obstetrics \& Gynecology, 2004, 103(6):1300-1307.

38. Shah NR and Bracken MB, A systematic review and meta-analysis of prospective studies on the association between maternal cigarette smoking and preterm delivery, American Journal of Obstetrics and Gynecology, 2000, 182(2):465-472.

39. Dewan $\mathrm{N}$ et al., The effects of smoking on birthweight-forgestational-age curves in teenage and adult primigravidae, Public Health, 2003, 117(1):31-35.

40. Delpisheh A et al., Population attributable risk for adverse pregnancy outcomes related to smoking in adolescents and adults, Public Health, 2007, 121(11):861-868.

41. Ventura SJ et al., Trends and variations in smoking during pregnancy and low birth weight: evidence from the birth certificate, 19902000, Pediatrics, 2003, 111(5, pt. 2):1176-1180.

42. DuPlessis HM et al., Adolescent pregnancy: understanding the impact of age and race on outcomes, Journal of Adolescent Health, 1997, 20(3):187-197. 
43. Pearl M et al., The relationship of neighborhood socioeconomic characteristics to birthweight among 5 ethnic groups in California, American Journal of Public Health, 2001, 91(11):1808-1814.

44. Pickett KE et al., Neighborhood socioeconomic status, maternal race and preterm delivery: a case-control study, Annals of Epidemiology, 2002, 12(6):410-418

45. Frederick IO et al., Pre-pregnancy body mass index, gestational weight gain, and other maternal characteristics in relation to infant birth weight, Maternal and Child Health Journal, 2007, 12(5): 557-567.

46. Schieve LA et al., Prepregnancy body mass index and pregnancy weight gain: associations with preterm delivery. The NMIHS Collaborative Study Group, Obstetrics \& Gynecology, 2000, 96(2):194-200.

47. Strang J and Story M, Adolescent nutrition, in: Brown JE, ed., Nutrition Through the Life Cycle, second ed., Belmont, CA: Thomson Wadsworth, 2005, pp. 325-352.

48. Nielsen JN et al., Interventions to improve diet and weight gain among pregnant adolescents and recommendations for future research, Journal of the American Dietetic Association, 2006, 106(11):1825-1840.

49. Sable MR and Herman AA, The relationship between prenatal health behavior advice and low birth weight, Public Health Reports, 1997, 112(4):332-339.
50. White DE et al., The content of prenatal care and its relationship to preterm birth in Alberta, Canada, Health Care for Women International, 2006, 27(9):777-792.

51. Whitlock EP et al., Behavioral counseling interventions in primary care to reduce risky/harmful alcohol use by adults: a summary of the evidence for the U.S. Preventive Services Task Force, Annals of Internal Medicine, 2004, 140(7):557-568.

52. Britt E et al., Motivational interviewing in health settings: a review, Patient Education and Counseling, 2004, 53(2):147-155.

53. Flynn L et al., Enhancing resource utilization among pregnant adolescents, Public Health Nursing, 2008, 25(2):140-148.

54. Crittenden KS et al., Smoking cessation processes in low-SES women: the impact of time-varying pregnancy status, health care messages, stress, and health concerns, Addictive Behaviors, 2007, 32(7):1347-1366.

\section{Acknowledgment}

This research was funded, in part, by The Robert Wood Johnson Health \& Society Scholars Program.

Author contact: partingt@uwm.edu 